\title{
Welchen Beitrag können die Wirtschaftswissenschaften leisten?
}

\author{
Seit zwei Jahren ist die Transformations- \\ forschung zur Nachhaltigkeit ein neues \\ interdisziplinäres Forschungsfeld. Auch die \\ Wirtschaftswissenschaften können mit ihren \\ Methoden und Theorien dazu beitragen. \\ Von Birgit Soete und Ulrich Petschow
}

\begin{abstract}
D er Wissenschaftliche Beirat der Bundesregierung Globale Umweltveränderungen (WBGU) versteht unter Transformationsforschung die wissenschaftliche Begleitung von gesellschaftlichen Such- und Lernprozessen zu einer nachhaltigen Gesellschaft. Deshalb sollte die Transformationsforschung neuen Typs interdisziplinär zwischen Ingenieur-, Natur- und Geistes- bzw. Sozialwissenschaften ausgerichtet sein und gleichzeitig transdisziplinär, um gesellschaftliche Partizipation an den Suchprozessen zu ermöglichen. Die Forschung selbst soll systemisches, reflexives und antizipatives Wissen erzeugen (WBGU 2011, S. 341).
\end{abstract}

\section{Transformationsforschung für Nachhaltigkeit}

Die bisherige historische Transformationsforschung hat gesellschaftliche Umbrüche beziehungsweise gesellschaftliche Transformationsprozesse wie die industrielle Revolution oder die politisch-wirtschaftliche Transformation der ehemaligen sozialistischen osteuropäischen Staaten sowie der ehemaligen Sowjetunion analysiert. Deutlich wurde, dass diese Transformationsprozesse ein Zusammenspiel von technischen, sozialen sowie institutionellen Innovationen, kulturellem Wertewandel und weiteren Promotoren sind. Eine Transformationsforschung neuen Typs sollte die historische Forschung mit der Zukunftsforschung und ihren Methoden kombinieren, um mögliche $\mathrm{Zu}$ künfte einer nachhaltigen Gesellschaft und Pfade dahin beschreiben zu können. Die Methoden der Zukunftsforschung wie die Szenarienentwicklung, die Delphi-Befragung oder Zukunftswerkstätten werden bereits zur Entwicklung solcher Pfade genutzt. Die interdisziplinäre Transformationsforschung sollte analysieren, was Auslöser und Treiber für gesellschaftliche Veränderungsprozesse sind und ob solche Prozesse gesellschaftlich gestaltbar sind.

Vor zwanzig Jahren hat sich die Politik in Rio de Janeiro auf der Internationalen Tagung Umwelt und Entwicklung (1992) auf das Leitbild einer nachhaltigen Entwicklung geeinigt. Seit dem wird darüber debattiert was man unter einer nachhaltigen Entwicklung verstehen will. Das Fortschreiten der globalen Umweltprobleme wie Klimawandel, Verlust der Biodiversität oder Verlust an fruchtbarem Boden macht die Dringlichkeit des gesellschaftlichen Handelns immer deutlicher. Gleichzeitig haben die globale Finanzmarkt- und Wirtschaftskrise von 2007 bis 2009 sowie die Euro-Krise von 2010 bis 2012 gezeigt, dass es durch die Globalisierung sowie die europäische Integration wirtschaftliche Interdependenzen gibt, die in den jeweiligen Krisen zu sozialen Verwerfungen geführt haben. Diese werden von den jeweiligen Gesellschaften nicht mehr akzeptiert. Seit den Krisenjahren wird, vor allem in der Politik, nach neuen Wegen gesucht, wie sozial- und umweltverträgliches Wirtschaften aussehen könnte. Transformationsforschung könnte hier einen Beitrag leisten und verschiedene mögliche Zukünfte sowie Pfade dahin aufzeigen. Welche technischen Möglichkeiten für umweltverträgliches Wirtschaften gibt es? Wie weit werden diese Technologien gesellschaftlich akzeptiert und integriert? Welche nationalen und internationalen Rahmenbedingungen brauchen diese Innovationen für die Diffusion? Wer wird Gewinner und wer Verlierer eines sozial- und umweltverträglichen Wirtschaftens sein? Sind die Wohlfahrtsgewinne eines Transformationsprozesses hinreichend groß, um die Verluste kompensieren zu können?

$\mathrm{Zu}$ all diesen Fragen können auch die Wirtschaftswissenschaften analytisch beitragen, wenn Ökonomien als Systeme verstanden werden, die nicht mechanistisch einer linearen Logik folgen. Transformationsprozesse können mit einer Kombination der Institutionenökonomik, evolutorischen Ökonomie, Innovationsökonomik, Spieltheorie und Verhaltensökonomik schon heute untersucht werden. Der Beitrag der Wirtschaftswissenschaften könnte in der Analyse von geeigneten Anreizmechanismen für Verhaltensänderungen, der institutionellen Voraussetzungen für wirtschaftlichen Wandel, des Marktdesigns für technologische und soziale Innovationen sowie der makroökonomischen Effekte des Strukturwandels liegen. Allerdings konzentriert sich die wirtschaftswissenschaftliche Forschung zur Nachhaltigkeit auf die Internalisierung externer Effekte und die Frage, wie weit Naturkapital durch menschengemachtes Kapital (Sachkapital, Humankapital, Sozialkapital) substituiert werden kann. Im Rahmen der ökologischen Ökonomie oder der Klimaökonomie wird über die lose Modellkoppelung von natürlichen Systemen mit ökonomischen Modellen versucht systemische Interaktionen abzubilden, aber auch hier bleiben viele Fragen offen. 


\section{Das Beispiel Energiesystemtransformation}

Der WBGU zeigt in seinem Gutachten „Gesellschaftsvertrag für eine große Transformation“ (2011), dass aus globaler Makrosicht eine Energiesystemtransformation zu einem dekarabonisierten Energiesystem technisch machbar ist, das heißt die entsprechenden Technologien existieren bereits und die notwendigen Investitionen können auch finanziert werden. Wenn man sich dann die Energiewende in Deutschland ansieht, muss man feststellen, dass das Energieziel, im Jahr 2050 den Strombedarf mit 80 Prozent aus Erneuerbaren Energien zu decken, definiert ist, aber der Weg dahin ist unklar und bislang wenig vorgedacht (BMWi, BMU 2010). Bereits 1991 hat Deutschland mit dem Stromeinspeisegesetz die erneuerbaren Energietechnologien gefördert. Mit dem Erneuerbaren Energien Gesetz (EEG) ab dem 1.4.2000 wurde eine Entwicklungsdynamik ausgelöst, sodass im Jahr 2011 der Anteil der Erneuerbaren Energien am Stromverbrauch bei gut 20 Prozent liegt (BMU 2012). In Kombination mit der Liberalisierung des Elektrizitätsmarktes ab dem Jahr 1998 und der Schaffung eines europäischen Energiebinnenmarktes hat das EEG als Markteinführungsprogramm seine Wirkung entfaltet. Allerdings wurde im Zuge der Liberalisierung ein Marktdesign geschaffen, das sich an den alten Erzeugungstechnologien sowie Marktstrukturen vertikal integrierter Unternehmen orientierte und die Integration neuer Technologien und neuer Wettbewerber vernachlässigte. Da die Kostenstruktur der erneuerbaren Energietechnologien eine andere ist, ist an der Strombörse der „Merit-Order-Effekt“ aufgetreten, in der Mittagszeit fällt der Strompreis erheblich durch die Solarenergie und damit die Strombeschaffungskosten für Großabnehmer (Musiol 2012). Gleichzeitig steigt die Differenz zwischen Marktpreis und Einspeisetarif.

Bisher wurde in der wirtschaftspolitischen Beratung zu wenig darüber nachgedacht, dass ein Systemumbau andere Rahmenbedingungen braucht als die partielle Betrachtung einzelner Marktsegmente. Hinzu kommt, dass Strom ein Gut ist, das netzgebunden und nicht lagerfähig ist. Der Systemumbau erfordert nicht nur die Einführung neuer Erzeugungstechnologien, sondern die Veränderung der Transportinfrastruktur und die Veränderung des Verbraucherverhaltens. Diese Komplexität ist bisher vernachlässigt worden. Notwendig wird die Entwicklung neuer Institutionen und Regelungen entlang der gesamten Wertschöpfungskette für Strom, damit die Energiewende technologisch, kosteneffizient, umwelt- und sozialverträglich gelingt.

\section{Transformation der Wirtschaftswissenschaften}

Zwar zeigen neue Veröffentlichungen (Buchholz et al. 2012; EFI 2013), dass die Komplexität der Energiewende und die Wechselbeziehungen des Elektrizitätsmarktes mit anderen Märkten, unter anderem dem Kapitalmarkt, erkannt werden, aber der Fokus der wirtschaftswissenschaftlichen Beratung liegt weiterhin auf der Etablierung von Instrumenten für die Angebotsseite. In dem aktuellen EFI-Gutachten (2013) werden die unterschiedlichen Dimensionen der Energiewende analysiert und Vorschläge für Instrumente entwickelt, die verschiedenen Externalitäten zu internalisieren, aber wie Verhaltensveränderungen auf der Nachfrageseite induziert werden, bleibt offen. Insgesamt bleiben die wirtschaftswissenschaftlichen Analysen weiter in der Grenzkostenbetrachtung verhaftet. Dadurch werden die Hemmnisse der institutionellen Rahmenbedingungen mit weitreichenden Verteilungsimplikationen ausgeblendet. Beispielsweise das seit Jahrzehnten diskutierte Eigentümer-Mieter-Dilemma im Bereich der Mietwohnungen.

An diesem aktuellen Beispiel wird deutlich, dass die wirtschaftswissenschaftliche Forschung und Beratung sich neuen Methoden und Theorien öffnen und problemadäquat ausdifferenzieren sollte. Die Transformationsforschung stellt eine Herausforderung für die Wirtschaftswissenschaften dar, aber sie kann auch ein Katalysator für diese werden, sodass sie sich selbst transformieren.

\section{Literatur}

Buchholz, W. et al. (2012): Die Zukunft der Energiemärkte. Ökonomische Analyse und Bewertung von Potenzialen und Handlungsmöglichkeiten, ifo Forschungsberichte 57, Studie in Kooperation mit der Forschungsstelle für Energiewirtschaft e.V. im Auftrag des BMWi, München.

BMU (2012): Entwicklung der erneuerbaren Energien in Deutschland im Jahr 2011. Grafiken und Tabellen, Berlin.

EFI - Expertenkommission Forschung und Innovation (2013): Jahresgutachten zu Forschung, Innovation und technologischer Leistungsfähigkeit Deutschlands 2013, Berlin.

BMWi, BMU (2010): Energiekonzept für eine umweltschonende, zuverlässige und bezahlbare Energieversorgung, Berlin.

Musiol, F. (2012): Erneuerbare Energien in Zahlen, in: Müller, T. (Hrsg.) 20 Jahre Recht der Erneuerbaren Energien, Schriften zum Umweltenergierecht, S. 123-128, Baden-Baden.

WBGU (2011): Welt im Wandel. Gesellschaftsvertrag für eine Große Transformation, Hauptgutachten, Berlin.

\section{AUTOREN + KONTAKT}

Dr. Birgit Soete ist Gastprofessorin für Volkswirtschaftslehre - Mikroökonomie an der Hochschule für Wirtschaft und Recht Berlin und IÖW Fellow.

E-Mail: birgit.soete@hwr-berlin.de

Ulrich Petschow ist Leiter des Forschungsfeldes Umweltökonomie und Umweltpolitik am Institut für ökologische Wirtschaftsforschung (IÖW).

IÖW, Potsdamer Str. 105, 10785 Berlin. Tel.: +49 $30884594-0$, E-Mail: ulrich.petschow@ioew.de, Website: www.ioew.de

Copyright (C) 2013, IÖW und oekom Verlag. Die Nutzung des Artikels ist Abonnenten von Ökologisches Wirtschaften vorbehalten. Nachdruck und Vervielfältigung des Artikels einschließlich Speicherung und Nutzung auf optischen und elektronischen Datenträgern nur mit Zustimmung der Redaktion von Ökologisches Wirtschaften (http://www.oekologischeswirtschaften.de). 